\title{
Corneal cell viability and structure after transcorneal freezing-thawing in the human cornea
}

This article was published in the following Dove Press journal:

Clinical Ophthalmology

14 May 2010

Number of times this article has been viewed

\author{
Joo Youn $\mathrm{Oh}^{1,2}$ \\ Hyun Ju Lee ${ }^{1,2}$ \\ Sang In Khwarg ${ }^{1,2}$ \\ Won Ryang Wee ${ }^{1,2}$ \\ 'Department of Ophthalmology, \\ Seoul National University College \\ of Medicine, Seoul, Korea; ${ }^{2}$ Seoul \\ Artificial Eye Center, Seoul National \\ University Hospital Clinical Research \\ Institute, Seoul, Korea
}

Purpose: Although cryotherapy has long been used to eradicate corneal lesions, there have been no reports of adverse effects of cryotherapy on human corneas. We performed this study to evaluate and characterize ultrastructural damage to the human cornea following the transcorneal freezing-and-thawing procedure.

Methods: Seven human donor corneas were randomly divided into three groups. 1, 2, and 3 repetitive freezing-and-thawing procedures were respectively applied to donor corneas in each group. A cryoprobe was cooled to $-80^{\circ} \mathrm{C}$, and placed on the anterior surface $1.5 \mathrm{~mm}$ central to the limbus for 3 seconds. Samples were then allowed to spontaneously defrost. A cornea without the treatment was used as a control. Samples were evaluated through hematoxylin \& eosin staining, TUNEL assay, and electron microscopy.

Results: After transcorneal cryoinjury, it was observed that corneal endothelial cells were lost and Descemet's membrane was denuded where the cryoprobe was applied. Corneal stromal cells were damaged, and the damage was more marked in the posterior stroma. The extent of damage increased with an increasing number of freezing-thawing repetitions. In contrast, corneal epithelial cells showed no cryo-induced damage, and Bowman's layer remained intact in all groups.

Conclusions: The susceptibility to transcorneal cryo-injury differed among the corneal layers; the corneal endothelium was most susceptible, and the epithelium was least susceptible. Caution would thus be advised in regard to the potential damage in corneal endothelium when treating patients with corneal lesions using transcorneal cryotherapy.

Keywords: apoptosis, cryotherapy, endothelium, keratocyte

\section{Introduction}

Cryodestruction of corneal lesions typically involves multiple repetitions of freezing and thawing using a cryoprobe placed transcorneally. ${ }^{1-5}$ However, in addition to destroying the lesion, previous reports have shown that transcorneal freezing can also damage the corneal endothelium in rabbits, resulting in clinically significant corneal edema. ${ }^{6,7}$ Corneal endothelial dysfunction is reversible in these animals, because extensive cellular division occurs at the margin of the wound, and damaged cells are completely replaced by new cells. ${ }^{8}$ In contrast, human corneal endothelium does not regenerate, and endothelial dysfunction can lead to irreversible corneal edema. ${ }^{9}$

Although the efficacy of cryotherapy in eradicating corneal lesions has been reported, ${ }^{1-5}$ the possibility of cryo-induced damage to cells and structures in human corneas has not been investigated. In the present study, we evaluated the ultrastructural changes in human cornea after repetitive transcorneal freezing-thawing $(\mathrm{F} / \mathrm{T})$ procedures.
Correspondence:Won Ryang Wee, Department of Ophthalmology, Seoul National University College of Medicine, 28 Yeongeon-dong, Jongno-gu, Seoul I I0-744, Korea Tel +82 2-2072-2435

Fax +82 27413187

Emailwrwee@snu.ac.kr 


\section{Materials and methods}

The study has been performed in accordance with the principles embodied in the Declaration of Helsinki.

\section{Transcorneal freezing}

Seven human donor corneas were used. The average donor age was 45 years (range 17 to 62 years). Donor corneas were preserved in Optisol GS at $4^{\circ} \mathrm{C}$ for 4 days before freezing. The corneas were randomly divided into three groups; 1,2 , and $3 \mathrm{~F} / \mathrm{T}$ were respectively applied to each group $(\mathrm{n}=2$ for each group). A single fresh, untreated donor cornea was used as a control. For freezing, a cryoprobe $(2.5 \mathrm{~mm}$ in diameter; ERBE Elektromedizin GmbH, Tuebingen, Germany) was cooled to $-80^{\circ} \mathrm{C}$ and then placed on the anterior surface of the peripheral cornea $1.5 \mathrm{~mm}$ central to the limbus, for 3 seconds. After freezing, a balanced salt solution was used to free the cryoprobe from the tissue, and the cornea was allowed to thaw naturally. The treatment was applied on six spots of the cornea around the limbus with the same distance apart. Donor corneas in the double and triple F/T groups were refrozen and thawed in a similar manner. The interval between F/T cycles was 1 minute.

\section{Histological examination}

Portions of the corneas from each group were sectioned and stained with hematoxylin \& eosin (H\&E) or subjected to terminal deoxynucleotidyl transferase-mediated nick end labeling (TUNEL) assay. TUNEL assay was performed using the ApopTag ${ }^{\circledR}$ Plus Fluorescein in situ apoptosis detection kit (Chemicon International, Billerica, MA, USA), according to the manufacturer's protocol. The H\&E-stained slides were observed under a light microscope (Olympus Optical Co., Ltd., Tokyo, Japan). TUNEL-positive cells were observed on TUNEL staining under a fluorescent microscope (BX-61, Olympus, Tokyo, Japan).

\section{Electron microscopy}

In order to evaluate the ultrastructural damage to corneal cells and collagen, the corneas from each group were dissected in pieces, $3 \times 3 \mathrm{~mm}$ from the center where the cryoprobe was employed, and prepared for electron microscopy. The posterior corneal surface, including the corneal endothelium, was evaluated by scanning electron microscopy (SEM), and the corneal section including stroma and keratocytes was examined by transmission electron microscopy (TEM). For SEM, samples were prefixed with $2.5 \%$ glutaraldehyde (PBS phosphate-buffered saline; $\mathrm{pH} 7.2$ ) at $4^{\circ} \mathrm{C}$ overnight. Following several washes in PBS, samples were kept in $1 \%$ osmium
tetraoxide-PBS for final fixation for 1 hour. Samples were then washed and dehydrated through serial dilutions of ethanol. Samples were mounted onto stubs, sputter-coated with gold by a Polaron SC-500 (VG Microtech, Sussex, UK), and finally examined with scanning electron microscopy (SEM) (JSM 1400; JEOL, Tokyo, Japan). For TEM, the corneas from each group were fixed with $2.5 \%$ glutaraldehyde PBS (pH 7.2) at $4^{\circ} \mathrm{C}$ overnight and post-fixed in $1 \%$ osmium tetroxide-PBS for one hour. Samples were then washed and dehydrated through serial dilutions of ethanol. Samples were mounted onto stubs, sputter-coated with gold by a Polaron SC-500 (VG Microtech), and finally examined with transmission electron microscopy (TEM) (JEM-1400; JEOL, Tokyo, Japan).

\section{Results}

H\&E staining showed a well demarcated area of denuded Descemet's membrane and endothelial damage in all groups, while the corneal epithelium and Bowman's layer were normal-appearing and intact (Figure 1). The disruption was most severe in the area where the cryoprobe was applied. Descemet's membrane was stripped, and corneal endothelial cells were lost. After three cycles of freeze-thaw, most of the corneal endothelial cells remaining near the probe site were TUNEL-positive on staining (Figure 2A).

Keratocytes, which are corneal stromal fibroblasts, were also damaged by cryotherapy as seen on TUNEL assay (Figure 2). TUNEL positivity was more remarkable in the posterior stromal keratocytes, compared to the anterior stromal keratocytes. Moreover, the extent of TUNEL

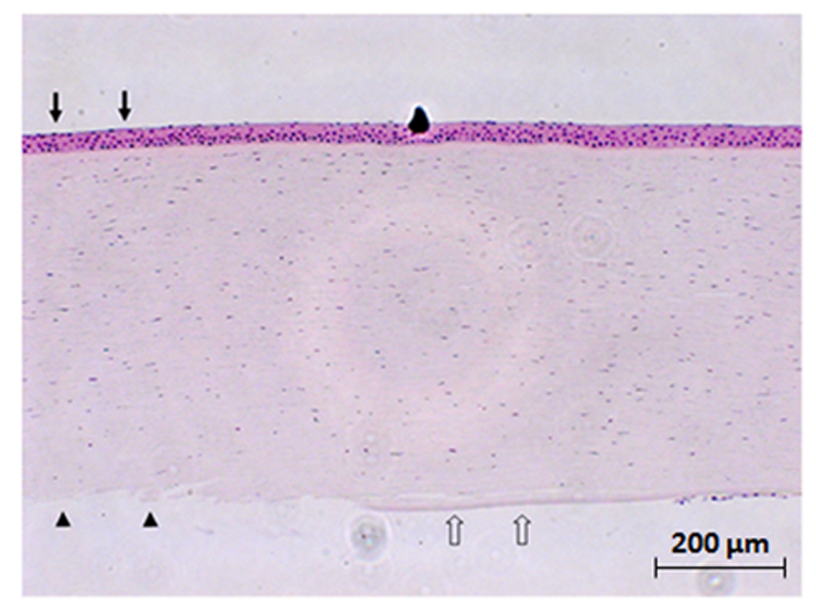

Figure I Hematoxylin \& eosin staining of the human cornea after three cycles of freezing-thawing. Descemet's membrane was denuded, and corneal endothelial cells were lost (arrowheads) where the cryoprobe was applied transcorneally (arrows). Adjacent to the probe application site, there was a denuded area with no corneal endothelial cells, although Descemet's membrane was present (empty arrows). Original magnification $\times 100$. 


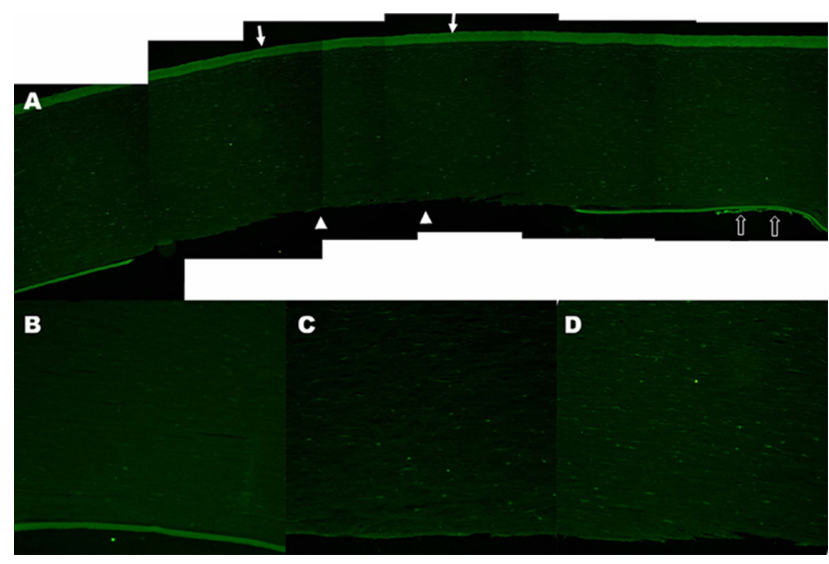

Figure 2 TUNEL assay of the human cornea. After three freezing-thawing (F/T) cycles, there was a well demarcated area of denuded Descemet's membrane with no endothelial cells (arrowheads) where the cryoprobe had been applied transcorneally (arrows) A) The surrounding corneal endothelial cells were TUNEL-positive (empty arrows) A) A few TUNEL-positive cells were also observed in the posterior corneal stroma after one F/T cycle B) and more TUNEL-positive cells were present in the cornea after two F/T cycles C) After $3 \mathrm{~F} / \mathrm{T}$ cycles, many TUNEL-positive cells were found throughout the whole thickness of the cornea $\mathbf{D}$ ) Descemet's membrane was intact in the single F/T cornea (B), while it was stripped off in the double and triple F/T corneas (C, D). Original magnification X50 (A), X100 (B, C, D).

positivity increased with the number of $\mathrm{F} / \mathrm{T}$ cycles applied to the cornea (Figures 2B-D).

SEM also demonstrated a loss of Descemet's membrane and disruption of the structure and continuity of the endothelial cell layer, as well as increasingly severe damage to the posterior surface with the increasing number of $\mathrm{F} / \mathrm{T}$ cycles (Figure 3). Apoptotic changes in the posterior stromal keratocytes were also observed by TEM (Figure 4).

\section{Discussion}

Several previous reports have demonstrated corneal endothelial damage following cryo-injury in rabbits and cats. ${ }^{6-8}$ Those studies used a large diameter cryoprobe to damage the central cornea, because the purpose of the experiments was to develop an in vivo model of corneal endothelial injury and recovery in animals. However, there have been no reports investigating the adverse effects of cryotherapy on human corneas. Considering that human corneal endothelial cells do not have mitotic activity and cannot regenerate, unlike their rabbit counterparts, ${ }^{6-9}$ endothelial damage by cryotherapy possibly leads to irreversible corneal edema in humans. In the present study, we applied a small diameter cryoprobe to the peripheral cornea and compared the damage among the three corneal cell layers: corneal epithelium, keratocytes, and endothelium. We found that the susceptibility to cryo-injury differed among the corneal layers. The corneal endothelium was most susceptible, and the epithelium was least

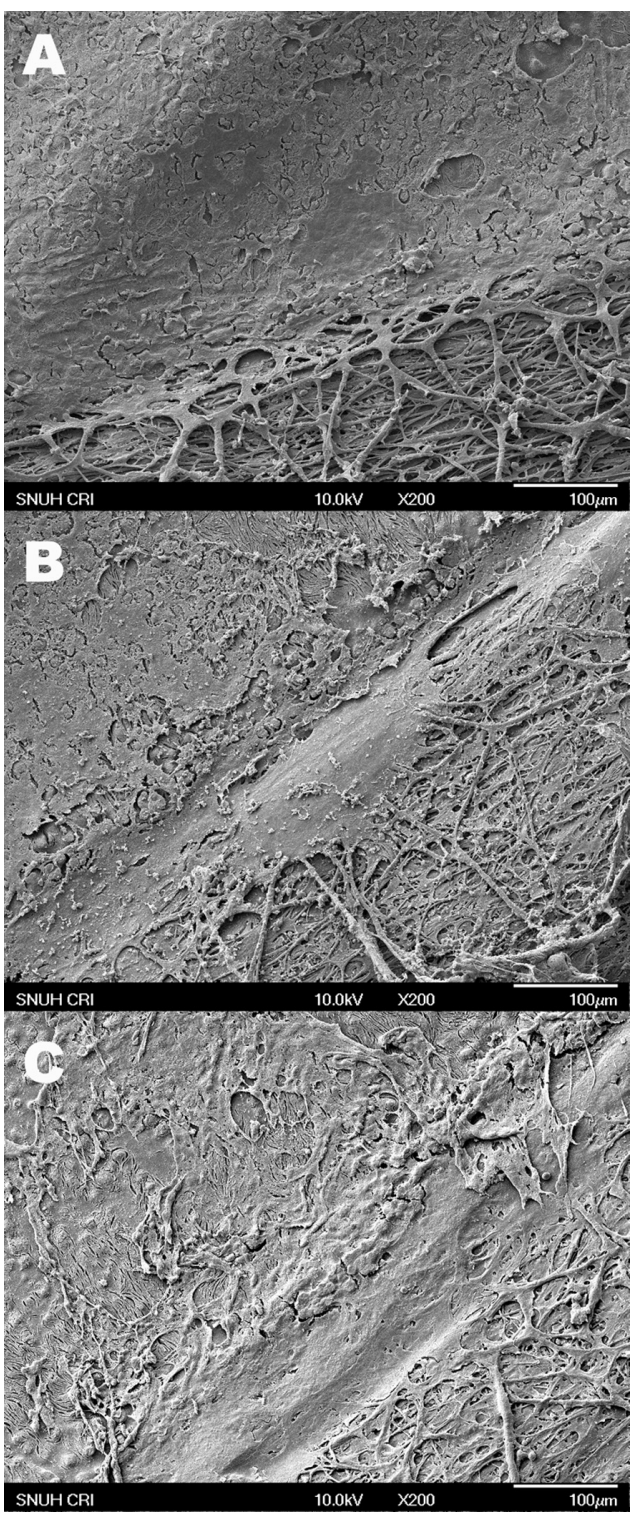

Figure 3 Scanning electron microscopic photographs of human corneal endothelium after I A) 2 B) and 3 C) freezing-thawing (F/T) cycles. A smooth surface of normal-appearing endothelial cells was observed adjacent to the bare cornea treated with one F/T cycle A) Corneal endothelial cell damage was more remarkable after 2 B) or 3 cycles of F/T. Descemet's membrane was stripped off where the cryoprobe was applied transcorneally, and the posterior stromal surface was exposed in all groups (right lower parts in A, B, and C) Original magnification X200.

susceptible. From this observation, it can be speculated that cryotherapy may cause an irreversible damage on human corneal endothelium. In this context, cryotherapy may not be used in ocular diseases related to the physiology of the corneal endothelium.

Also, it was observed that TUNEL positivity was highest at the center of the frozen volume where the cryoprobe was applied, and repetition of the F/T cycle induced greater cellular damage. Moreover, keratocytes in the posterior stroma were more severely damaged by cryo-injury than 


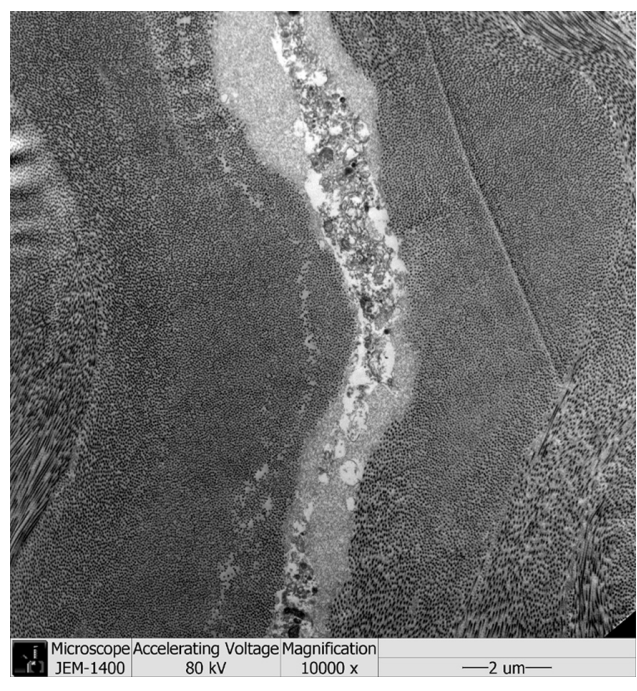

Figure 4 Transmission electron microscopic photographs of the stroma in human cornea after three cycles of freezing-thawing. Keratocyte nuclei are fragmented into several small chromatin masses, and apoptotic bodies are present. Original magnification $10,000 X$.

those in the anterior stroma were. This might have been due to the fact that the interval between repetitive $\mathrm{F} / \mathrm{T}$ cycles was longer in the posterior stroma than it was in the anterior stroma. Because the cryo-injury was applied transcorneally from the anterior surface to the posterior surface, the posterior stroma was the last portion of the cornea to be frozen during each cycle, and the first to be thawed. Thus, the interval between $\mathrm{F} / \mathrm{T}$ cycles was most delayed in the posterior part of the cornea. The delay in repetition allows time for vascular stasis that can enhance the destructive effect of the second cycle. ${ }^{10}$ Otherwise, the posterior keratocytes might be more susceptible to cryo-injury than the anterior keratocytes are.

The present study has several limitations. Firstly, we applied cryo-injury to the corneas after they were removed from the eyeballs. This might not appropriately simulate the in vivo situation where the corneal endothelium is in contact with the aqueous humor in the anterior chamber. The aqueous humor might exert some buffering or protective effect on the corneal endothelium during transcorneal freezing and thawing. Secondly, we did not perform the functional assay with regard to endothelial permeability and corneal thickness after cryotherapy. Thirdly, we did not evaluate cryo-injury damage to the cornea as it relates to varying cooling rates, temperature, and F/T duration and interval. Further study is necessary to determine the optimal protocol for cryotherapy and to maximize the elimination of corneal pathology while minimizing corneal toxicity. Lastly, it is possible that the wound healing process of corneal epithelium might be disrupted by cryotherapy although the epithelium and Bowman's layer remained intact immediately after the injury.

In conclusion, we found that human cornea was susceptible to transcorneal cryo-injury and the susceptibility differed among the corneal layers. The corneal endothelium was most susceptible, while the epithelium was least susceptible. We advise caution in the use of cryotherapy for the patients with ocular diseases related to the physiology of the corneal endothelium.

\section{Disclosures}

No authors have any financial/conflicting interests to disclose.

\section{References}

1. Klüppel M, Reinhard T, Sundmacher R, Daicker B. Therapy of advanced amoeba keratitis with keratoplasty à chaud and adjuvant cryotherapy. Ophthalmologe. 1997;94:99-103.

2. Finger PT. "Finger-tip" cryotherapy probes: treatment of squamous and melanocytic conjunctival neoplasia. Br J Ophthalmol. 2005;89: 942-945.

3. Panda A, Sharma N, Sen S. Massive corneal and conjunctival squamous cell carcinoma. Ophthalmic Surg Lasers. 2000;31:71-72.

4. Peksayar G, Soyturk MK, Demiryont M. Long-term results of cryotherapy on malignant epithelial tumors of the conjunctiva. Am J Ophthalmol. 1989;107:337-340.

5. Fraunfelder FW. Liquid nitrogen cryotherapy of advancing wavelike epitheliopathy. Cornea. 2006;25:196-198.

6. Minkowski JS, Bartels SP, Delori FC, Lee SR, Kenyon KR, Neufeld AH. Corneal endothelial function and structure following cryo-injury in the rabbit. Invest Ophthalmol Vis Sci. 1984;25:1416-1425.

7. Buco P, Van Horn DL, Schutten WH, Cohen K. Effects of transcorneal freezing on protein content of aqueous humor and intraocular temperature in rabbit and cat. Invest Ophthalmol Vis Sci. 1978;17: 1199-1202.

8. Van Horn DL, Sendele DD, Seideman S, Buco PJ. Regenerative capacity of the corneal endothelium in rabbit and cat. Invest Ophthalmol Vis Sci. 1977; 16:597-613.

9. Liesegang TJ. The response of the corneal endothelium to intraocular surgery. Refract Corneal Surg. 1991;7:81-86.

10. Baust JG, Gage AA. Progress towards optimization of cryosurgery. Technol Cancer Res Treat. 2004;3:95-101.

\section{Clinical Ophthalmology}

\section{Publish your work in this journal}

Clinical Ophthalmology is an international, peer-reviewed journal covering all subspecialties within ophthalmology. Key topics include: Optometry; Visual science; Pharmacology and drug therapy in eye diseases; Basic Sciences; Primary and Secondary eye care; Patient Safety and Quality of Care Improvements. This journal is indexed on
PubMed Central and CAS, and is the official journal of The Society of Clinical Ophthalmology (SCO). The manuscript management system is completely online and includes a very quick and fair peer-review system, which is all easy to use. Visit http://www.dovepress.com/ testimonials.php to read real quotes from published authors. 